\section{FRI0394 THE ROLE OF D-DIMER TEST AS A SCREENING TOOL FOR VENOUS THROMBOEMBOLISM IN PATIENTS WITH SYSTEMIC LUPUS ERYTHEMATOSUS AS COMPARED TO MATCHED CONTROL SUBJECTS}

Y.J. Oh, E.H. Park, J.W. Park, Y.W. Song, E.B. Lee. Division of Rheumatology, Department of Internal Medicine, Seoul National University College of Medicine, Seoul, Korea, Republic Of

Background: d-dimer test is widely used as a screening tool for venous thromboembolisms (VTE). Meanwhile, d-dimer can increase in various conditions including severe infection, and inflammation. However, it has been rarely reported whether d-dimer test is useful for screening of VTE in systemic lupus erythematosus (SLE) patients.

Objectives: We evaluated the role of d-dimer test as a screening tool for VTE in patients with SLE, compared to age-, and sex-matched non-autoimmune disease subjects.

Methods: In this retrospective cohort study, a total of 283 SLE patients and 1132 age-, and sex-matched control subjects (those who had no rheumatic diseases) who underwent d-dimer test as a screening test for VTE were enrolled at Seoul National University Hospital between January 2000 and July 2017. VTE was defined to be present when a thromboembolism was proven in imaging studies which included computed tomography, lung perfusion scan or duplex ultrasonography. Predictive value of d-dimer test for VTE was compared between SLE patients and control subjects by calculating area under the curves (AUC) in receiver operating characteristics (ROC) curves of d-dimer test. Finally, the usefulness of d-dimer test was evaluated in different subsets of SLE patients by analysing ROC curves.

Results: The mean (SD) age of the 283 SLE patients was 36.8 (13.5) years and that of 1132 control subjects was $38.2(12.8)$ years. The mean (SD) plasma level of d-dimer was $2262.1(3794.5) \mathrm{ng} / \mathrm{ml}$ in SLE patients, while it was 1087.5 $(5063.1) \mathrm{ng} / \mathrm{ml}$ in the control group $(\mathrm{p}<0.001)$. The incidence of VTE was significantly higher in SLE patients than the controls $(12.7 \%$ vs. $5.8 \%, p<0.001)$. When the cut-off value of d-dimer test was set to $500 \mathrm{ng} / \mathrm{ml}$, the AUC for VTE was only 0.614 in SLE patients, while it was 0.891 in the control group, suggesting that $d-$ dimer test may not be useful as a screening tool for VTE in SLE patients. When the SLE patients were divided according to the presence of antiphospholipid antibodies (APS Abs), the AUC value for VTE was 0.788 in patients who didn't have APS Abs but it was only 0.556 in patients who had APS Abs.

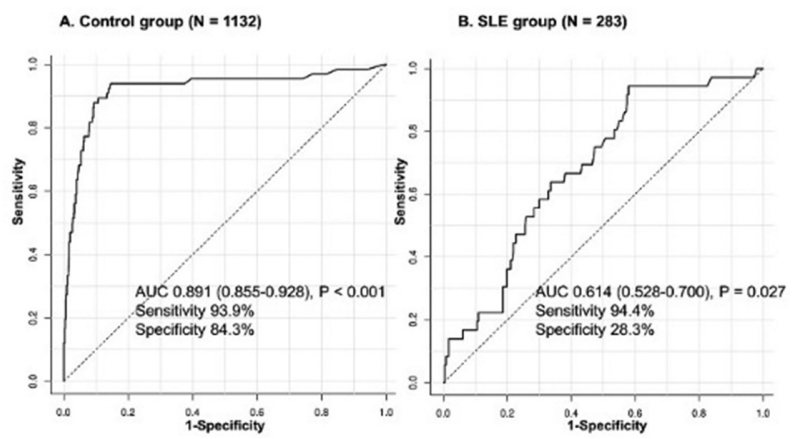

Abstract FRI0394 - Figure 1. ROC curves in control and SLE group (cut-off value of ddimer $=500 \mathrm{ng} / \mathrm{ml}$ )

Conclusions: d-dimer test cannot predict VTE in SLE patients as accurately as in general population. In SLE patients, d-dimer's diagnostic capability for VTE is even lower in the presence of APS Abs.

\section{REFERENCES:}

[1] Chung WS, Lin CL, Chang SN, et al. Systemic lupus erythematosus increases the risks of deep vein thrombosis and pulmonary embolism: a nationwide cohort study. J Thromb Haemost 2014;12:452-8.

[2] Avina-Zubieta JA, Vostretsova K, De Vera MA, et al. The risk of pulmonary embolism and deep venous thrombosis in systemic lupus erythematosus: A general population-based study. Semin Arthritis Rheum 2015;45:195201.

[3] Kristoffersen AH, Ajzner E, Rogic D, et al. Is D-dimer used according to clinical algorithms in the diagnostic work-up of patients with suspicion of venous thromboembolism? A study in six European countries. Thromb Res 2016;142:1-7.

[4] Ahlehoff O, Wu JJ, Raunso J, et al. Cutaneous lupus erythematosus and the risk of deep venous thrombosis and pulmonary embolism: A Danish nationwide cohort study. Lupus 2017;26:1435-9.
[5] Wells PS, Ihaddadene R, Reilly A, et al. Diagnosis of Venous Thromboembolism: 20 Years of Progress. Ann Intern Med 2018;168:131-40.

Disclosure of Interest: None declared

DOI: 10.1136/annrheumdis-2018-eular.2250

\section{FRI0395 SLE DISEASE ACTIVITY INDEX GLUCOCORTICOID INDEX (SLEDAI-2KG) IDENTIFIES MORE RESPONDERS THAN SLEDAI-2K}

Z. Touma ${ }^{1}$, D. Gladman ${ }^{1}$, J. Su², N.M. Anderson ${ }^{2}$, M.B. Urowitz ${ }^{1} .{ }^{1}$ Internal Medicine, Division Of Rheumatology, UNIVERSITY OF TORONTO; ${ }^{2}$ Internal Medicine, Division Of Rheumatology, Krembil Research Institute, University Health Network, Toronto, Canada

Background: Systemic Lupus Erythematosus Disease Activity Index-2000 (SLE DAI-2K) is one of the most commonly used disease activity indices in clinical practice and research but this index doesn't account for severity within each descriptor. Moreover, in clinical trials, the use of standard of care (SoC), which includes glucocorticoid (GC) often confounds trial results.

We developed and validated a novel lupus disease activity index, SLEDAI-2K GC (SLEDAI-2KG), that describes disease activity while accounting for GC dose SLEDAI-2KG has the same descriptors as SLEDAI-2K in addition to a new descriptor "GC" with different weight scores based on the dose of GC. Furthermore, SLEDAI-2KG has a low administration burden and a simple scoring system similar to SLEDAI-2K.

Objectives: We aimed to compare the performance of SLEDAI-2K and SGI in identifying responders in response to $\mathrm{SoC}$

Methods: Patients have been followed prospectively according to a standard protocol between January 2011 and January 2014, at a single lupus centre, with active disease (SLEDAI-2K $\geq 6$ ), on prednisone $\geq 10 \mathrm{mg} /$ day, and with follow up visits within 5-24 months were studied. Treatment was determined based on the judgment of the treating rheumatologist.

Response to SoC therapy, at first follow up visit, was assessed by SLEDAl- $2 \mathrm{~K}$ and SLEDAI-2KG. Responders were defined based on the decrease in SLEDAI$2 \mathrm{~K}$ and SGI score by $\geq 4$. The performance of SLEDAI-2K and SGI was also compared using different cut-off points; 5, 6 and 7 . Descriptive analysis was used. Results: 111 patients met the inclusion criteria of the study and were further analysed. Patients' characteristics are represented in table 1.

SLEDAI-2KG identified more responders at 6 months (94\% vs. $84 \%)$ and at 12 months $(92 \%$ vs. $76 \%$ ) compared to SLEDAI-2K by cut off of 4 . SLEDAI-2KG also identified more responders with cut off points 5,6 and 7 (table 2).

Abstract FRI0395 - Table 1. Patient Characteristics

\begin{tabular}{lc}
\hline Variables & Total $(\mathrm{n}=111)$ \\
\hline Sex (female) & $98(88.3 \%)$ \\
Age at baseline (mean $\pm S D)$ & $35.75 \pm 11.51$ \\
SLE duration at baseline (mean $\pm S D)$ & $9.02 \pm 7.74$ \\
Ethnicity & $13(11.7 \%)$ \\
Asian & $23(20.7 \%)$ \\
Black & $51(45.9 \%)$ \\
Caucasian & $24(21.6 \%)$ \\
Others & \\
Months from baseline to 1 st follow-up (mean $\pm S D)$ & $7.68 \pm 2.95$ \\
SLEDAI-2K at baseline (mean $\pm S D)$ & $12.39 \pm 6.03$ \\
Prednisone dose at baseline (mg/day) (mean $\pm S D)$ & $22.94 \pm 14.19$ \\
SLEDAI-2KG at baseline (mean $\pm S D)$ & $17.48 \pm 6.78$ \\
SLEDAI-2K at 1 st follow-up & $8.08 \pm 6.04$ \\
Prednisone dose at 1 st follow-up (mg/day) (mean $\pm S D)$ & $15.23 \pm 10.94$ \\
SLEDAI-2KG at 1 st follow-up & $12.67 \pm 6.98$
\end{tabular}

Abstract FRI0395 - Table 2. Responders by SLEDAI-2K and SLEDAI2KG in 111 patients

\begin{tabular}{lcccccccc}
\hline Indices & \multicolumn{3}{c}{$\begin{array}{c}\text { Percentage of responders at } \\
6 \text { months }\end{array}$} & \multicolumn{3}{c}{$\begin{array}{c}\text { Percentage of responders at } \\
12 \text { months }\end{array}$} \\
\hline & $\geq 4$ & $\geq 5$ & $\geq 6$ & $\geq 7$ & $\geq 4$ & $\geq 5$ & $\geq 6$ & $\geq 7$ \\
\hline SLEDAI-2K & $84 \%$ & $68 \%$ & $68 \%$ & $59 \%$ & $76 \%$ & $57 \%$ & $55 \%$ & $44 \%$ \\
SLEDAI-2KG & $94 \%$ & $88 \%$ & $83 \%$ & $79 \%$ & $92 \%$ & $80 \%$ & $70 \%$ & $62 \%$ \\
Additional Responders & $10 \%$ & $20 \%$ & $15 \%$ & $20 \%$ & $16 \%$ & $23 \%$ & $15 \%$ & $18 \%$ \\
\hline
\end{tabular}

Conclusions: The novel index, SLEDAI-2KG, is superior to SLEDAI-2K in identifying responders at 6 and 12 months accounting for steroid dose and thus adjusting for severity within each descriptor of SLEDAI-2K. SLEDAI-2KG has the ability to enhance analyses in clinical trials to differentiate between responders on minimal and moderate/large doses of GC. 
Disclosure of Interest: Z. Touma Grant/research support from: GlaxoSmithKline, D. Gladman: None declared, J. Su: None declared, N. Anderson: None declared, M. Urowitz Grant/research support from: GlaxoSmithKline DOI: 10.1136/annrheumdis-2018-eular.5519

FRIDAY, 15 JUNE 2018

\section{Systemic sclerosis, myositis and related syndromes - etiology, pathogenesis and animal models}

\section{FRI0396 THE ATM KINASE AND PTEN, DRIVE MYOFIBROBLASTS DIFFERENTIATION BY ACTIVATING THE TGFB AUTOCRINE LOOP}

S. Xu ${ }^{1}$, I. Papaioannou ${ }^{1}$, M. Ponticos ${ }^{1}$, C. Denton ${ }^{1}$, A. Wells ${ }^{2}$, E. Renzoni ${ }^{2}$, D. Abraham ${ }^{1} .{ }^{1}$ Centre for Rheumatology and Connective Tissue Diseases, UCL Medical School (Royal Free Campus), London, UK; ${ }^{2}$ Interstitial Lung Disease Unit, NHLI, Royal Brompton Hospital/Imperial College, London, UK

Background: Pulmonary fibrosis is a major cause of mortality in scleroderma (SSc) and Idiopathic Pulmonary Fibrosis (IPF). Fibrosis is driven by Inappropriate myofibroblast differentiation and persistence. Understanding this process, is vita for developing an effective treatment. Angiotensin II, is implicated in fibroblast activation in the heart and kidney, through interactions with growth factors (e.g. EGF and TGF $\beta$ ).

Objectives: We examined the role of Angiotensin II in myofibroblast activation in the lung.

Methods: Lung fibroblasts were isolated from SSc, IPF, or control patient lungs (6 each). Fibroblasts were also cultured from PTEN null and wild-type mice. Protein expression after angiotensin II treatment (Angll) was investigated by western blotting. Myofibroblast differentiation and function was assayed through the contraction of 3D collagen gels and scratch migration assays. The signalling pathways involved were dissected using specific inhibitors: PI3-kinase/AKT (wortmannin, LY294002), TGFß (1d11 neutralising antibody, SB431542 ALK5 inhibitor) Ataxia-Telangiectasia Mutated (ATM - Ku55933), Angll (Losartan). Results: SSc and IPF lung fibroblasts showed increased AKT phosphorylation and suppressed PTEN expression $(\mathrm{p}<0.05)$. Their phenotype was more myofibroblast-like, with higher $\alpha$ SMA expression $(p<0.05)$, increased collagen gel contraction (control; $207 \pm 14$ vs SSc; $93 \pm 15$ vs IPF $91 \pm 21, \mathrm{p}<0.05$ ), and enhanced migratory capacity $(p<0.05)$. PTEN-null fibroblasts showed a similar phenotype. Angll treatment activated AKT, suppressed PTEN and induced myofibroblast differentiation in normal lung fibroblasts. In both Angll-treated and PTEN lung fibro blasts AKT activation required the ATM kinase. Inhibition of AKT either with PI3K or ATM inhibitor abrogated these effects. The increased expression of Myofibro blast-related genes after Angll treatment, was also blocked by inhibition of TGF $\beta$ with a neutralising antibody or an ALK5 inhibitor. AKT phosphorylation on the other hand was only partially blocked was partially blocked by TGF $\beta$ inhibition. Conclusions: Our data demonstrate for the first time that Angll signals via the ATM kinase, which together with PTEN suppression are essential for the activation of AKT by Angll. Angll promotes myofibroblast differentiation, by stimulating the fibroblast TGF $\beta$ autocrine loop through AKT. Our data shows that activation of AKT through ATM and PTEN, may serve as the molecular link between pulmonary hypertension and lung fibrosis in fibrotic diseases.

Acknowledgements: Arthritis Research UK, Royal Free Hospital Charity and Scleroderma Research UK.

Disclosure of Interest: None declared

DOI: 10.1136/annrheumdis-2018-eular.5224

\section{FRI0397 PECULIAR EXPRESSION OF AUTOPHAGY BIOMARKERS IN NECROTIZING AUTOIMMUNE MYOPATHY MUSCLE}

M. Giannini ${ }^{1}$, F. Girolamo ${ }^{2}$, A. Amati ${ }^{2}$, A. Lia ${ }^{2}$, L. Serlenga ${ }^{3}$, D. D'Abbicco ${ }^{4}$,

M. Tampoia ${ }^{5}$, F. lannone ${ }^{1} .{ }^{1}$ Rheumatology Unit, University of Bari; ${ }^{2}$ D.S.M.B.N.O.

S. Neurology, Bari; ${ }^{3}$ Ospedale L. Bonomo, Andria, BT; ${ }^{4}$ Marinaccio General

Surgery, Bari; ${ }^{5}$ University Clinical Pathology Laboratory, Bari, Italy

Background: Immune mediated necrotizing myopathy (IMNM) is a recently recognised pathology within the spectrum of idiopathic inflammatory myopathies (IIMs). Specific autoantibodies and the response to immunosuppressants aid to make the diagnosis and suggest immune-mediated pathogenesis, although histopathological features are not specific for IMNM. Autophagy and ubiquitin-proteasome system are two interacting systems by which dysfunctional cellular components are degraded in the cell. Their dysregulation, in sporadic Inclusion
Body Myositis (sIBM), seems to be responsible for the protein aggregates. The autophagy dysfunction in IMNM was not widely investigated.

Objectives: To investigate autophagy marker expression, macrophages localization and accumulation of misfolded proteins in non-necrotic fibres of IMNM muscle in comparison with Dermatomyositis (DM), Polymyositis (PM) and sIBM.

Methods: Among 52 IIMs diagnosed from January 2015 to June 2017, we reviewed muscle biopsies and stored sera. Six subjects were included in the IMNM group, characterised by many necrotic muscle fibres, regenerating muscle fibres and no significant inflammation despite of numerous but scattered macrophages removing necrotic muscle fibres. Two patients had anti-signal recognition particle (SRP) autoantibodies, two patients anti-3-hydroxy-3-methylglutaryl-coenzyme A reductase (HMGCR), the others tested negative for specific autoantibod ies. All IMNM patients had a positive response to immunosuppressants. Muscle sections were immunolabelled with the following antigens: ubiquitin, autophagy markers LC3b, p62 (a receptor of autophagy), TDP-43 (a marker of ubiquitinated proteic inclusions), SMI31 and SMI310 (Phosphorylated Neurofilaments), CD31 (endothelial cell marker), C5b-9 (membrane attack complex), CD4 (T-helper lymphocytes), CD8 (T-suppressor lymphocytes), CD68 (macrophages), CD20 (Blymphocytes), CD56 (NK lymphocytes and regenerating muscle fibres), MHC I MHC II. Quantitative results were compared among IMNM $(n=6), D M(n=4)$, sIBM $(n=4), P M(n=5)$ and healthy controls $(n=4)$

Results: In IMNM, inflammation was mild compared with DM, PM, sIBM, and consisted in sporadic endomysial and/or perivascular cells CD68+. Skeletal muscle fibres (SMFs) containing LC3b+puncta were significantly higher in IMNM and IBMs than in DM or PM. In all IMNM, the greater proportion of LC3b+puncta was localised in CD56 +fibres (figure 1), instead, sIBM showed a high number of LC3b +puncta in vacuolated SMFs with low expression of CD56 +SMFs. As expected, P62 and SMI31 aggregates were significantly higher in sIBM than in the othe IIMs, even if, also in IMNM, there were moderate p62 accumulations and a little proportion of SMFs stained by SMI31. Finally, in IMNM there was the highest number of ubiquitin + SMFs.

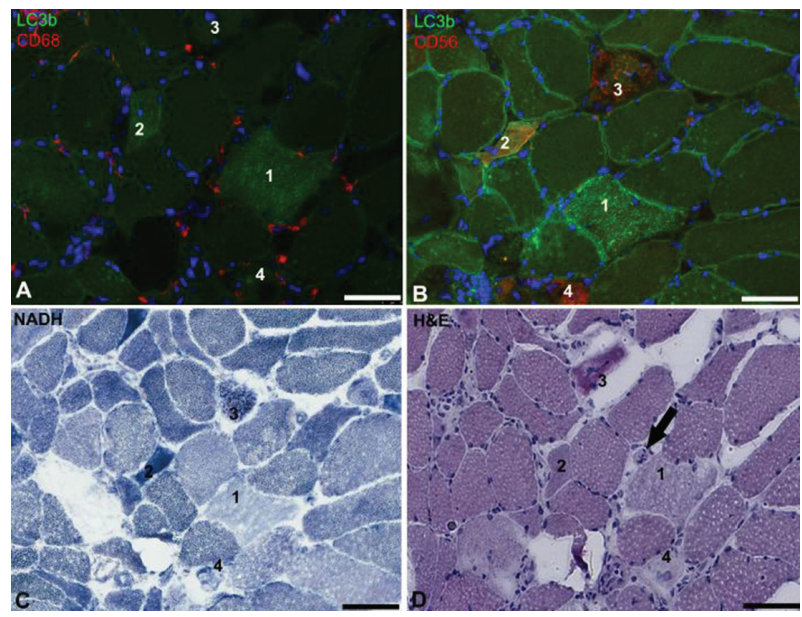

Conclusions: These findings suggest an involvement of cellular clearance sys tems in the pathophysiology of IMNM similarly to sIBM. Nevertheless, LC3b +puncta in regenerating fibres can be considered a peculiar biomarker in IMNM. Further studies of larger cohort of patients are needed to better define IMNM. Disclosure of Interest: None declared DOI: 10.1136/annrheumdis-2018-eular.2530

\section{FRI0398 \\ SL-401, A NOVEL TARGETED THERAPY DIRECTED TO THE INTERLEUKIN-3 RECEPTOR (CD123), KILLS PLASMACYTOID DENDRITIC CELLS FROM SYSTEMIC SCLEROSISPATIENTS}

M.-D. Ah Kioon ${ }^{1}$, R. Lindsay ${ }^{2}$, J. Chen ${ }^{2}$, J. Gordon ${ }^{3}$, R. Spiera ${ }^{3}$, F. Barrat ${ }^{1}$, C. Brooks ${ }^{2} .{ }^{1}$ Autoimmunity and Inflammation Program, Hospital for Special Surgery Research Institute; ${ }^{2}$ RandD, Stemline Therapeutics; ${ }^{3}$ Scleroderma and Vasculitis Center, Hospital for Special Surgery, New York, USA

Background: SL-401 is a novel biologic targeted therapy directed to the interleu kin-3 receptor (CD123). SL-401 is comprised of human IL-3 recombinantly fused to a truncated diphtheria toxin (DT) payload engineered such that IL-3 replaces the native DT receptor-binding domain. In this way, the IL-3 domain of SL-401 directs the cytotoxic DT payload to cells expressing CD123. Upon internalisation, $\mathrm{SL}-401$ irreversibly inhibits protein synthesis and induces apoptosis of the target cell. 